Is social inhibition of return due to action co-representation?

Mark A. Atkinson ${ }^{1}$, Andrew Simpson ${ }^{1}$, Paul A. Skarratt ${ }^{2}$, Geoff G. Cole ${ }^{1}$

1. Centre for Brain Science, University of Essex.

2. Department of Psychology, University of Hull.

Running head: Social IOR and action co-representation.

Key words: Social IOR- Joint action - Social attention - biological motion objects

Submitted: 28/03/2014

Word count: 7611

Address for correspondence

\author{
Mark A Atkinson \\ Centre for Brain Science \\ University of Essex \\ Wivenhoe Park \\ Colchester \\ CO4 3 SQ \\ E-mail: matkinb@essex.ac.uk \\ Tel: 01206872575 \\ Fax: 01206873801
}




\begin{abstract}
When two individuals alternate reaching responses to visual targets presented on a shared workspace, one individual is slower to respond to targets occupying the same position as their partner's previous response. This phenomenon is thought to be due to processes that inhibit the initiation of a movement to a location recently acted upon. However, two distinct forms of the inhibition account have been posited, one based on inhibition of an action, the other based on inhibition of an action and location. Furthermore, an additional recent explanation suggests the phenomenon is due to mechanisms that give rise to action congruency effects. Thus the three different theories differ in the degree to which action co-representation plays a role in the effect. The aim of the present work was to examine these competing accounts. Three experiments demonstrated that when identical actions are made, the effect is modulated by the configuration of the visual stimuli acted upon and the perceptual demands of the task. In addition, when the co-actors perform different actions to the same target, the effect is still observed. These findings support the hypothesis that this particular joint action phenomenon is generated via social cues that induce locationbased inhibition of return rather than being due to shared motor co-representations.
\end{abstract}




\section{General Introduction}

The past decade has seen increasing interest in the effect of interpersonal interaction on human cognition (Atmaca, Sebanz, \& Knoblich, 2011; Atmaca, Sebanz, Prinz, \& Knoblich, 2008; Frischen, Loach, \& Tipper, 2009; Schuch \& Tipper, 2007; Tsai, Sebanz, \& Knoblich, 2011; Welsh, et al., 2005). Such research has revealed novel insights concerning cognitive processes that have previously been studied with individuals, including visual attention and motor performance. Focusing upon the latter behaviors, recent interest in 'joint action' is in part due to the acknowledgement that many everyday human visuomotor behaviors involve interaction with others, or acting in the close presence of others.

Joint action work has most often been placed within the context of models that link action and perception (Hommel, 2009; Jeannerod \& Frak, 1999; Knoblich \& Sebanz, 2006; Prinz, 1997). The basis of these models is that rather than being separate, perception and action share cognitive representations. It follows that when two or more people act together, the observation of one individual's action by another activates the motor system of the observer. Co-representation of perceived and performed actions has received much support (see Blakemore \& Frith, 2005; Wilson \& Knoblich, 2005 for reviews). Sebanz, Knoblich and Prinz (2003) demonstrated this phenomenon using the Simon spatial compatibility task (Simon, 1970). When this task is performed alone, a discrimination of a stimulus is made that has two dimensions. The dimension to be discriminated (for example, a color) is non-spatial but the other dimension is spatial (for example, an arrow). Participants make discriminations using buttons placed spatially so that they can be spatially congruent 
or incongruent with the stimuli. Typically, congruent responses are faster than those that are incongruent. Sebanz, et al. found that this occurred when two individuals were each responsible for making a single discrimination response but did not do so when a single person performed one such response, i.e., in the absence of a co-actor. The so-called Social Simon Effect (SSE) has been interpreted as evidence that individuals represent the actions and/or task of another, as if they were their own (Dolk, et al., 2011; Sebanz, Knoblich, \& Prinz, 2005b; Sebanz, Knoblich, Prinz, \& Wascher, 2006 but see Dolk et al., 2011).

Another example of a joint action effect that has generated a considerable amount of interest, and central to the present work, concerns social inhibition of return (social IOR; in which the actions of one individual can lead to inhibition in an observer. In the basic experiment, participants sit facing each other and take turns to respond to targets presented on a flat workspace positioned between them (Figure 1). Results typically show that an individual is slower to initiate a response to a stimulus presented at the same location as their partner's previous response. Welsh et al. explained the effect with a combination of the action-perception models referred to above, the mirror neuron system, and inhibition of return (IOR; Posner \& Cohen, 1984). Specifically, since inhibition is a known consequence of action (e.g., Howard, Lupáñez, \& Tipper, 1999; Tremblay, Welsh, \& Elliott, 2005; Welsh \& Pratt, 2006), the authors argued that the observer may inhibit an action based on one previously observed. In other words, the same inhibitory processes are evoked when a participant observes another person act on a location and when they act upon the location themselves. Furthermore, Welsh et al. posited that the mirror neuron system (Fogassi, Ferrari, Gesierich, Rozzi, Chersi, \& Rizzolatti, 2005; Rizzolatti \& Craighero, 2004) 
could play a role in this process. Mirror neurons are found in parietal and premotor areas of macaque monkeys and recently in a network of cortical and subcortical areas in humans (Fabbri-Destro \& Rizzolatti, 2008; Mukamel, Ekstrom, Kaplan, Iacoboni, \& Fried, 2010). These neurons are activated both during the performance and execution of a specific action and may be responsible for directly creating motor representations when an action is observed (di Pellegrino, Fadiga, Fogassi, Gallese, \& Rizzolatti, 1992; Filimon, Nelson, \& Hagler, 2007; Gallese, Fadiga, Fogassi, \& Rizzolatti, 1996). The final aspect of Welsh et al.'s theory concerns IOR. It is been well-established that after shifting attention to a location, a person will inhibit responses to stimuli appearing at the same location (Kingstone \& Pratt, 1999; Klein, 2000; Posner \& Cohen, 1984; Rafal, Calabresi, Brennan, \& Sciolto, 1989). Although the precise mechanisms underlying IOR are debated, it is widely thought that attentional and oculomotor processes are slowed in reorienting to previously cued regions of space. This phenomenon is manifested by a delay in manual response time (RT) to respond to targets that are previously cued, relative to those that are uncued. Thus, Welsh et al. argued that the above processes act together to generate the basic effect.

In later work, Welsh and colleagues have provided further evidence that action corepresentation can influence social IOR (Welsh, McDougall, \& Weeks, 2009). In a modification to the basic social IOR paradigm, participants now sat side-by-side rather than facing one another. As before, each took turns to reach out to one of two targets appearing on a tabletop. One target could occur on the right of the participant sitting on the right, another target could appear on the left of the participant sitting on the left, and a third target could appear at a position located between the two and was 
used by both participants. In accordance with the usual social IOR finding, results showed that responses were relatively slow when a reaching response was made to the same target as their partner's previous response. However, participants were also slower when making the same egocentric response as their partner. In other words, a participant sitting on the right would be slower to respond to their right hand target when their partner had also just made a rightward response. Thus, a partner's arm action movement appeared to be represented, rather than simply their response location.

The action co-representation account of social IOR does not however concur with recent work examining whether social IOR represents other aspects of actions. Cole, Skarratt, and Billing (2012; see also Ondobaka, de Lange, Wiemers, NewmanNorlund, \& Bekkering, 2012) undertook a variant of the basic social IOR procedure in which participants reached to a location and either performed the same end-point action as their partner (e.g., both writing a digit with a pencil) or performed a different end-point action (e.g., one writes a digit, the other erases a digit). This was partly motivated by evidence showing that perceptuo-motor representations are sensitive to action goals and end states (e.g. Fogassi, et al., 2005; Iacoboni, et al., 2005). Cole et al. however showed that the magnitude of social IOR was independent of action goal compatibility. Although it could be argued that the mechanisms that cause social IOR do represent actions but not their goals, these findings fit better with the alternative inhibitory account of the basic effect. Cole et al. (2012) suggested that when an individual responds to a spatial location this will direct an observer's perceptuo-motor processing to that location. Consequently, IOR will be generated resulting in slower RTs to targets appearing at the responded-to location. In effect, the target and the 
subsequent arm reach elicits the same orienting response as does the central or peripheral cue in the standard IOR paradigm.

Ondobaka et al. (2012) have recently presented a further account of the basic arm movement phenomenon in which the effect was placed within the context of action congruency mechanisms. Performing an action is known to facilitate the initiation of a similar action in an observer. For instance, Liepelt, von Cramon, and Brass, (2008; see also, Brass, Bekkering, Wohlschlager, \& Prinz, 2000; Kilner, Paulignan, \& Blakemore, 2003) presented photographs of a hand that had a target number placed over the image. Observers were required to discriminate the target and make a response by lifting either their index or middle finger. The important manipulation was that the hand on the photograph had either its index or middle finger raised. Results showed that when the target required the middle finger to be raised reponses were faster if the depicted hand also had the middle finger raised. The same effect occurred for the index finger. With respect to the present effect, Ondobaka et al. argued that when a participant sees their co-actor perform a particular action this facilitates the same action performed themselves within an egocentric framework. For instance, if a co-actor sees their partner reach to their right this facilitates a rightward reach when they themselves are required to reach to their right on the next trial. Indeed, as well as describing social IOR in terms of slowed responses, the effect can also be described as an effect in which RTs are shorter when a co-actor performs the same action as their partner. This description, favoured by Ondobaka et al. is is therefore a pure co-representation account, where only action congruency mechanisms are implicated. 
The principal aim of the present work was to directly examine whether social IOR depends on the representation of an observed action, as suggested by Welsh et al. and Ondobaka et al. or can be accounted for by orienting mechanisms representing spatial locations. In three experiments participant pairs performed variants of the standard social IOR procedure in which we manipulated aspects of the stimuli as well as the actions each one made in relation to their partner. In Experiments 1 and 2 the actions performed by each participant were kept constant with only visual aspects of the stimuli being manipulated. If social IOR is caused by action co-representation mechanisms, no modulation of the basic effect should occur because observed and performed actions were identical across the two levels of the action factor (i.e., same location as previous response or different location). In direct contrast to Experiments 1 and 2, Experiment 3 manipulated each partner's actions but kept the stimuli identical. In this case, if the action co-representation account holds, then these manipulations should modulate the effect because co-actors sometimes performed different actions.

\section{Experiment 1}

Experiment 1 tested the action co-representation accounts of social IOR by having participants make identical responses to one another. As set out above, no modulation of the basic effect should be observed under such conditions. Furthermore, whilst the action factor was kept constant we manipulated the visual factors known to influence

IOR. Specifically, we examined whether the perceptual grouping of stimuli influenced the effect. It is well established that preattentive segmentation processes can influence 
the allocation of attention, such that representations can be based upon objects as well as spatial location (Duncan, 1984). Studies of IOR have also demonstrated that inhibition can be object-based. IOR can spread from one spatial location to another if both locations form part of the same object (Jordan \& Tipper, 1999) and can travel with an object as it moves from one location to another (Jordan \& Tipper, 1998; Tipper, Jordan, \& Weaver, 1999).

Following the standard social IOR procedure, pairs of participants alternated single responses to left and right targets on a shared work surface. Each 'placeholder' contained either a single pair of possible targets, a pair of separated targets embedded within a rectangular object, a single pair that moved between two locations, or a pair separated without the grouping object (Figure 2). To reiterate, if action corepresentation is the mechanism by which social IOR occurs, there should be no change in the magnitude of the basic effect across the perceptual grouping conditions because actions were kept constant.

\subsection{Method}

\subsubsection{Participants}

Twenty-four participants (age range 20 - 25 years old, 15 females) completed the study in return for course credit. All participants were right-handed and naive to the purpose of the experiment. 


\subsubsection{Apparatus}

A Pentium PC running custom software controlled the display of stimuli and the recording of response data. Stimuli were presented on a 22-inch LCD touchscreen monitor that was set into the surface of a table. The monitor screen, lay with its surface lying $740 \mathrm{~mm}$ from the floor and $240 \mathrm{~mm}$ from each participant's 'home' button. These buttons were serial PC button boxes, which participants rested upon when not responding. Home buttons were released when actions where initiated on a trial, providing a starting point for response times.

\subsubsection{Stimuli}

The stimuli were presented on the touch screen. A central black fixation cross $(0.4$ $\left.\mathrm{cd} / \mathrm{m}^{2}\right)$ was set against a uniform white $\left(73.7 \mathrm{~cd} / \mathrm{m}^{2}\right)$ background. Response positions were black squares $\left(0.4 \mathrm{~cd} / \mathrm{m}^{2}\right)$ and grouping objects were dark grey $\left(28 \mathrm{~cd} / \mathrm{m}^{2}\right)$. Position squares became targets by illuminating to white $\left(73.7 \mathrm{~cd} / \mathrm{m}^{2}\right)$. Figure 2 shows the target stimuli in each condition. In the condition where participants responded to the same pair of single targets ('same static' condition), a single target was presented to the left and right of fixation at a distance of $175 \mathrm{~mm}$ as measured from their middle. In the condition with pairs of displaced targets ('displaced ungrouped' condition) four squares were located on the edge of an imaginary rectangle whose longest side was $350 \mathrm{~mm}$ and shortest side was $110 \mathrm{~mm}$, again with the centre $175 \mathrm{~mm}$ from fixation. In the condition where pairs of targets were grouped within the same object ('displaced grouped' condition) a rectangle surrounded the targets, measuring 140mm long and $22 \mathrm{~mm}$ wide. Finally, in the condition where a set of single targets moved towards each participant ('single moving' condition), the target positions were the same as they were for the separate and same conditions. 


\subsubsection{Design}

A 2 x 4 repeated measures design was employed. The first factor ('target location') varied the left/right position of a participant's target in relation to their partner's last target response. This factor had two levels, 'same' and 'different', which were presented pseudorandomly throughout each block of trials. The second factor ('display type') varied the perceptual aspects of the stimuli as shown in Figure 2. This had four levels; 'single static', 'displaced grouped', 'displaced ungrouped' and 'single moving'. The levels of this factor were blocked and counterbalanced according to a Latin square design.

\subsubsection{Procedure}

Participants were asked to use their preferred hand to rest upon and depress their home button whilst maintaining fixation centrally. Upon seeing each target occur, they alternated responses rapidly and accurately by reaching with their preferred hand and tapping the target on the touchscreen. The first target was responded to by Participant A, the second by Participant $\mathrm{B}$, and so on until all the trials were completed. The custom software generated pseudorandom target presentations according to the following constraints: First, the number of targets presented to each participant at left and right locations was equated. The number of targets presented at the same side, relative to the previous trial, in comparison with those presented to the opposite side was also equal. No target appeared in the same left or right location on more than four occasions sequentially. Each block consisted of 209 trials, with the 
first trial serving only to begin the response sequence and therefore not included in the analysis. This left 52 same trials and 52 different trials for Participant A and the same for Participant B, yielding a total of 836 trials in the experiment.

\subsection{Results and Discussion}

Reaction times in excess of $1000 \mathrm{~ms}$ or shorter than $100 \mathrm{~ms}$ were excluded from further analysis. This resulted in the omission of $5.48 \%$ of responses. No localization errors occurred.

Figure 3 shows the mean (RTs) for each of the four conditions. A $2 \times 4$ repeated measures ANOVA was performed with display type (single static, displaced grouped, displaced ungrouped and single moving) and target location (same or different) as the two factors. This revealed a main effect of display type $(F(3,23)=4.08, p=.010$, $\left.\eta_{p}{ }^{2}=.150\right)$ as well as a main effect of location $\left(\mathrm{F}(1,23)=61.3, \mathrm{p}<.001, \eta_{p}{ }^{2}=.727\right)$, this latter effect being indicative of social IOR. There was also a significant interaction between location and display type $\left(\mathrm{F}(1,23)=25.1, \mathrm{p}<.001, \eta_{p}{ }^{2}=.522\right)$. Bonferoni-corrected t-tests were used to identify whether social IOR occurred with each type of display. These revealed a significant effect when participants acted upon a single static object $(\mathrm{t}(23)=7.43, \mathrm{p}<.001, \mathrm{~d}=1.51$, two-tailed $)$, displaced grouped objects $(\mathrm{t}(23)=3.35 \mathrm{p}=.003, \mathrm{~d}=.668$, two-tailed $)$, and a single moving object $(\mathrm{t}(23)$ $=2.82 \mathrm{p}=.010, \mathrm{~d}=.576$, two-tailed). When participants acted upon displaced ungrouped objects however, no significant effect of location was observed $(\mathrm{t}(23)=$ $.568, \mathrm{p}=.576, \mathrm{~d}=.115$, two-tailed). 
These results show that the magnitude of social IOR varied according to the perceptual groupings of the stimuli. Of particular note was the presence of the effect when the two observers acted upon the same object, and its absence when they acted upon different objects (i.e., the displaced ungrouped condition). That these perceptual manipulations modulated the social IOR effect when all responses generating the effect were identical challenges Ondobaka et al.'s view that the effect is due to action congruency mechanisms. The results do not however rule out the location inhibition account of Welsh et al. (2005); responses were not slowed to a location adjacent to that just responded to unless grouped with it. Indeed the results are consistent with a visuomotor inhibition account of social IOR.

Overall the results of Experiment 1 indicate that social IOR codes both objectbased and space-based representations. These results are consistent with the proposal that similar orienting mechanisms subserve both social IOR and classical IOR effects. Manipulations of these mechanisms can modulate the social IOR effect despite participant's observation of the same act that is performed.

\section{Experiment 2}

Experiment 1 showed that, contrary to the pure co-representaion account of the phenomenon (Ondobaka et al. (2012), social IOR can be modulated by perceptual grouping processes. This occurred independently of the kind of action that participants performed. Nonetheless, it is still possible that the effect occurs as a result 
of shared perceptuo-motor representations, but these representations may be sensitive to action effects and stimuli, including the presence of objects (e.g., Johnson-Frey, et al., 2003; Villiger, Chandrasekharan, \& Welsh, 2011).

In Experiment 2, whilst co-actors again performed the same actions as each other (i.e., reach to the same location, different location), they undertook either a standard detection task, where a reaching response was made to a single target left or right of fixation or, alternatively, a discrimination task in which participants responded to a square of one colour and ignored a square of a different colour. Not only does this allow us to again assess Ondobaka et al.'s action congruency account (because actions are kept constant), it also allows us to assess whether the effect shares a fundamental characteristic with IOR. A well-established feature of IOR is that it is generally much smaller, if present at all, when a discrimination is made between two stimuli, as opposed to detection or localization (Kwak \& Egeth, 1992; Tanaka \& Shimojo, 1996; Terry, Valdes, \& Neill, 1994). Thus, if social IOR is based on action corepresentation, an effect of similar magnitude should be observed irrespective of the task demands (i.e., the actions being the same in both tasks). In contrast, if it is due to processes more akin to classical IOR, as suggested by the data from Experiment 1, the inhibitory effect ought to be smaller or absent when targets are discriminated than when they are merely localized.

In addition, if attentional/oculomotor processes drive social IOR then the magnitude of the effect should be related to classical IOR (i.e., the original form of IOR). Such a relationship has been reported by Welsh et al. (2007) who showed that 
the size of social IOR correlates with that of non-social IOR. Nonetheless, a question remains as to whether this relationship was due to participants representing their own and another's goal-directed action in a functionally equivalent way or alternatively, whether the observation of another's action is an attention-capturing event, in the manner of a transient cue as presented in a classic IOR paradigm. The current experiment therefore also tested whether a relationship was present between individual IOR and social IOR. To that end, participants performed both a social IOR procedure as well as the standard "three box" precueing paradigm requiring only a single Go-No go response (i.e., Posner \& Cohen, 1984). This was to determine whether a relationship was present when no goal-directed actions were performed in the individual IOR paradigm.

\subsection{Method}

\subsubsection{Participants}

24 participants (22 females, 18-26 years old) were recruited in exchange for course credit. None of the participants completed the previous experiment or any other social IOR study reported here. All were right-handed.

\subsubsection{Apparatus}

All apparatus for the social IOR tasks were as in Experiment 1. The IOR task was completed on Apple eMac computers with 17" monitor displays. Stimuli were presented and responses recorded using Superlab (Cedrus systems). 


\subsubsection{Stimuli}

For the social IOR tasks, all aspects were as reported in Experiment 1 with the following exceptions. In the discrimination task, the two response positions were a green $\left(50.6 \mathrm{~cd} / \mathrm{m}^{2}\right)$ and red box $\left(10.6 \mathrm{~cd} / \mathrm{m}^{2}\right)$, presented simultaneously. For the standard IOR task, participants sat $400 \mathrm{~mm}$ from the display. A green fixation dot was presented on a uniform black background. This fixation dot was surrounded by grey cue box $\left(28 \mathrm{~cd} / \mathrm{m}^{2)}\right.$ measuring $3.44^{\circ}$ horizontally and $1.71^{\circ}$ vertically. This cue box illuminated to white following the presentation of the peripheral cue. Target locations were light grey boxes $\left(4.72^{\circ}\right.$ horizontally and $2.86^{\circ}$ vertically) each positioned $2.86^{\circ}$ horizontally from fixation as measured from the centre. These were surrounded by dark grey target cue boxes (positioned $0.43^{\circ}$ horizontally and vertically around the target box), which illuminated white to cue the target locations. Targets themselves were Gabor patches (sinusoidal gratings enveloped by a Gaussian; 2 cycles/deg, $7.9^{\circ}$ $\times 7.9^{\circ}$ ) and were presented in the centre of the peripheral boxes.

\subsubsection{Design}

The experiment employed a $2 \times 3$ repeated measures design. The first factor ('target location') again manipulated the location of a participant's target relative to their partner's previous response (i.e., 'same' and 'different'). These were pseudorandomly presented within each block of trials as described in Experiment 1. The second factor varied the particular kind of IOR task that participants performed ('task type'). This had three levels, 'social IOR localization', 'social IOR discrimination' and 'individual 
IOR'. The levels of this factor were blocked and their presentation order fully counterbalanced.

\subsubsection{Procedure}

The procedure in the social IOR tasks were as described for Experiment 1, with the following exceptions. In the social IOR discrimination task, participants were either asked to respond to a specific target (red) and to ignore the target located on the opposite side of fixation (green). These appeared simultaneously against a white background for the $1200 \mathrm{~ms}$ response period, following a 350ms fixation period. In the IOR detection task, participants were asked to fixate centrally throughout the procedure. Upon the appearance of the Gabor target in each trial, they were to respond as quickly and as accurately as possible by pressing the space bar. Each trial began with the presentation of the blank fixation display for $1000 \mathrm{~ms}$ followed by either the left or right peripheral cue for $100 \mathrm{~ms}$. The central fixation cue then occurred for $750 \mathrm{~ms}$ after which the target appeared. This remained visible until a response was given. Participants completed a single block of 104 experimental trials and 24 catch trials in the IOR detection task.

\subsection{Results and Discussion}

Four participants were omitted from the analysis. Two because $50 \%$ or more of responses failed to register with the touchscreen and two because $50 \%$ or more of responses were greater than $1000 \mathrm{~ms}$ in the IOR detection task. Of the remaining participants, approximately $6.78 \%$ of the data was omitted due to responses that failed 
to register the touch screen, as well as those that were initiated prior to $100 \mathrm{~ms}$ or after $1000 \mathrm{~ms}$ elapsing from the presentation of targets. No localization errors occurred within the remaining responses in any of the tasks.

Mean RTs for the three conditions are shown in Figure 4. A 2 × 2 repeated measures ANOVA was performed on the two social IOR task with task type (social IOR localization or social IOR discrimination) and target location (same or different) as the factors. This revealed a main effect of target location $(\mathrm{F}(1,19)=17.9, \mathrm{p}<.001$, $\left.\eta_{p}{ }^{2}=.485\right)$ but no main effect for task type $\left(\mathrm{F}(1,19)=1.22, \mathrm{p}=.091, \eta_{p}{ }^{2}=.143\right)$, again confirming the presence of social IOR. There was however, a significant interaction between target location and task type, $\left(\mathrm{F}(1,19)=77.5, \mathrm{p}<.001, \eta_{p}^{2}=.803\right)$. As before, Bonferoni-corrected $t$-tests were used to identify whether inhibition occurred at each level of task type. These revealed a significant social IOR effect when participants performed the social IOR localization task, $(\mathrm{t}(19)=7.66, \mathrm{p}<.001, \mathrm{~d}=1.71$, twotailed). In the social IOR discrimination task, the test for location revealed a significant effect in the opposite direction $(\mathrm{t}(19)=2.61, \mathrm{p}=.017, \mathrm{~d}=0.58$, two-tailed) providing no evidence for social IOR in this condition. The individual IOR task was analyzed separately using a paired-samples t-test. An IOR effect was also present in this task $(\mathrm{t}(19)=3.55 \mathrm{p}=.002, \mathrm{~d}=0.79$, two-tailed $)$. A difference score between novel and return locations was calculated for each participant for the two tasks where IOR was observed. This was to assess whether performance in the IOR task ( $M=20.9$, SD $=26.4)$ was correlated with performance in the social IOR localization task $(\mathrm{M}=$ 62.3, SD = 36.4). A Pearson's bivariate correlation confirmed a significant relationship between these two difference scores $(r=.372, \mathrm{p}<0.05)$. 
The results of Experiment 2 are consistent with those of Experiment 1; social IOR can be modulated according to visual and task demands when action responses remain the same. Inhibition was evident in terms of individual IOR and social IOR localization responses, but not in the social IOR discrimination task. Furthermore, the findings also support the prediction that there would be a relationship between performance on the individual IOR and social IOR localization tasks. As such, these results once again cast doubt on the idea that social IOR is mediated by action congruency mechanisms (i.e., Ondobaka et al. 2012) since no effect was observed in the discrimination condition when an action was congruent with respect to the previous response. However, the fact that the effect shares a characteristic with IOR, that is, its reduced magnitude when a person is required to perform a discrimination, further support the orienting account based on the inhibition of objects and regions of space.

\section{Experiment 3}

In Experiments 1 and 2 the actions performed by participants were the same (i.e., same location or different location with respect to the previous response) with only the stimuli and/or task demands being different. In Experiment 3 this was reversed so that the stimuli were the same across all conditions but the actions performed were different. The congruency of observed and performed actions was manipulated such that on half of the trials the co-actors performed the same actions while on the other half they performed different actions. If shared perceptuo-motor representations such as action congruency are responsible for social IOR, the effect should be diminished if 
the kinematics of the observed action differs from those performed by the observer (Brass, Bekkering, \& Prinz, 2001; Calvo-Merino, Glaser, Grezes, Passingham, \& Haggard, 2005). Experiment 3 therefore examined whether social IOR would occur when the observed and performed actions were different. To that end, participants again took part in a social IOR procedure, this time comprising four blocks of trials. In one block both participants reached for the target, in a second block both pointed to the target, in a third Participant A reached whilst Participant B pointed, and in the fourth Participant A pointed whilst Participant B reached. An action co-representation account of social IOR predicts that no basic effect will occur when co-actors perform very different actions (i.e., one points, the other reaches out). By contrast the orienting account predicts that social IOR will occur in all conditions; a co-actor will cue spatial locations by either pointing or reaching.

\subsection{Method}

\subsubsection{Participants}

The study recruited a sample of 40 different right-handed undergraduates in return for course credit (33 females, age 20 - 35 years). None had participated in the previous experiments.

\subsubsection{Apparatus}

All aspects of the stimuli and apparatus were as reported in Experiment 1. Because the different action conditions would give rise to large differences in sensory signals, these were controlled by limiting all visible information to a central portion. In line 
with Skarratt et al. (2010) and Welsh et al. (2005) we therefore masked the peripheral transients generated by the reaching action with a barrier placed between the two participants. The barrier included an aperture through which the initiation of a partners' reaching action or their point response could be observed, while occluding the peripheral target locations and the faces of partners.

\subsubsection{Stimuli}

The stimuli used were as in the grouped condition of Experiment 1. This set of stimuli allowed each participant to respond to the same target object but at the different locations necessitated by the use of the control barrier.

\subsubsection{Design}

A $2 \times 2 \times 2$ design was implemented, with three within-participant factors. The first factor (target location) again consisted of two levels (same and different). The second factor (action) had two levels corresponding to each type of action (point, reach). The third factor (congruency) varied whether participants performed the same or different actions. This factor had two levels (congruent, incongruent). The levels of action and congruency were blocked into four experimental conditions and presented such that each level appeared in two of the four blocks. These four blocks were fully counterbalanced.

\subsubsection{Procedure}


This was as reported previously with the following exceptions. When a participant responded in the pointing condition, they were instructed to release the home button and perform a pointing gesture by extending a single finger in the direction of the target, returning their hand to the home button. They were requested to complete this gesture as quickly and as accurately as possible. Moreover, they were asked to ensure that the gesture was kept low and without extending their arm toward the target so that it would be visible through the barrier window.

\subsection{Results and Discussion}

Outlier RTs were excluded as in Experiments 1 and 2, and resulted in the removal of $3.2 \%$ of the data.

The mean RT data are shown in Figure 5, and were analyzed in a $2 \times 2 \times 2$ ANOVA. Repeated measures factors consisted of target location (same and different), action (point and reach) and congruency (same or different). The main effect of target location was significant, $\mathrm{F}(1,39)=10.5 \mathrm{p}<.002, \eta_{p}{ }^{2}=.213$, again showing the presence of social IOR. There was also a main effect of action, $\mathrm{F}(1,39)=9.35, \mathrm{p}=$ $.004, \eta_{p}^{2}=.193$, however that of congruency was not significant, $\mathrm{F}(1,39)=3.74, \mathrm{p}=$ $.060, \eta_{p}^{2}=.088$. Surprisingly, the lack of a main effect for congruency showed that performing the same action as another did not affect the speed of action preparation. There was however, a difference in the time to prepare pointing and reaching actions. Importantly, there was also no interaction between the three factors, $F(1,39)=.369 p$ $=.547, \eta_{p}^{2}=.009$ and all other interactions were not significant $(\mathrm{p}>.500)$. This 
showed that the presence of the social IOR effect was resistant to whether a pointing or reaching action was performed and to whether these actions matched or mismatched those of a coactor.

These results show that social IOR occurred irrespective of whether co-actors performed the same or a different action to that of their partner. This reveals that participants do not need to perform the same manual action as a co-actor in order to inhibit subsequent movements to the same location. Even when an observer reaches rather than points (or vice versa), social IOR is still observed. In other words, these data suggest that simply directing another person's attention to a location, irrespective of how this is achieved, is enough to induce inhibition in the observer. These findings thus show that shared perceptual motor representations are not necessary to observe social IOR. Rather, it appears that any attention-capturing social cue is sufficient to delay a subsequent response to the same location.

The current findings also replicate those of Skarratt et al. (2010) who similarly employed barriers to restrict peripheral information. Additionally, the present data replicate the observation that these effects are small in comparison with either the basic effect in non-restricted conditions or the same procedure when peripheral transients are masked but participants have access to both gaze and action cues from their partner. This is most likely due to the lack of low-level sensory transients visible to participants in these conditions, despite the fact that the social information is sufficient to detect where a partner has responded. 
The present experiment demonstrates that the emergence of social IOR does not require co-actors to share an action representation. They do however suggest a trend towards overall shorter response times when participants make the same action (e.g., they both pointed). A wealth of evidence suggests that observing congruent actions facilitates the performance of subsequent actions (e.g. Brass, Bekkering, Wohlschläger, \& Prinz, 2000; Stürmer, Aschersleben, \& Prinz, 2000). Despite this slight facilitation for performing the same action, the effect failed to reach significance and inhibitory mechanisms were not modulated by the nature of the action performed. This pattern of data indicates that the present joint action paradigm is sensitive enough to reveal differences in reaction time on the basis of observed and performed kinematics, if they are present.

\section{General Discussion}

A number of studies have now demonstrated that people are slower to respond to stimuli that have been previously responded to by another individual. Two related accounts have suggested that this 'social IOR' effect (Skarratt et al., 2010) is due to co-representing an observed action. The present work investigated whether any form of action co-representation is needed for social IOR to occur. In Experiment 1, participants alternated responses to target stimuli that differed in their perceptual groupings. In Experiment 2 the task demands differed such that participants were required to localize or discriminate targets. Importantly, in both experiments the response actions remained the same while the perceptual and task demands were varied. In Experiment 3, this pattern was reversed such that the stimuli remained the 
same but now the actions were manipulated. The findings of all three experiments were consistent; when perceptual parameters were varied but action parameters were kept constant, social IOR was modulated (consistent with effects reported in the classical IOR literature). When, by contrast, perceptual parameters were kept constant, but response actions were manipulated, there was no modulation of the social IOR effect. Instead, social IOR was present, and to the same magnitude, when the observed and performed actions differed.

The results do not therefore support the action co-representation account of Ondobaka et al. (2012) and the strictest form of the Welsh et al. $(2005 ; 2007)$ account. Ondobaka et al. (2012) put forward what may be called a 'pure' co-representation account, suggesting that the effect is due to participants representing a physical imitative movement within an egocentric framework. For example, if one participant makes a reaching response to their left an observer will be quicker to initiate the same action themselves (i.e., reach to their own left). The current data are not compatible with this particular action co-representation account. In Experiments 1 and 2 co-actors performed identical actions to each other, that is, they reached to their right or left. Despite this, the size of the social IOR effect was modulated according to perceptual and task conditions. Perhaps more critical for this account were the results of Experiment 3. Even when participants performed two distinctly different goal directed actions (pointed or reached) social IOR was still observed. 
The action co-representation account of Welsh et al. $(2005 ; 2007)$ states that since inhibition is a known consequence of a prior action (e.g., Howard, et al., 1999; Tremblay, et al., 2005; Welsh \& Pratt, 2006), and that observing an action is functionally equivalent to performing it oneself (Sebanz, Knoblich, \& Prinz, 2003; Sebanz, Knoblich, \& Prinz, 2005a) it therefore follows that inhibition is induced when a participant observes another person acting on a location. According to this account, action co-representation does not facilitate the same physical movement but instead triggers the same spatial inhibitory mechanisms associated with performing two consecutive actions. However in the present Experiment 3, inhibition associated with a location was induced in an observer even though the observed co-actor had not acted upon it, only pointed to it. With the strictest interpretation of Welsh et al.'s account then, our data do not support their view that the inhibition of a location necessarily requires an action to be made upon it. However, it is possible that any observed action is sufficient to activate an inhibitory action-location map providing it indicates an object or location. In this case, a pointing response may therefore be functionally equivalent to a reaching response in activating such a map. At present this remains only an intriguing possibility, however one which suggests that pointing responses, like reaching and eye gaze direction belong to a class of social cues that orient observers to regions in space. It may of course be debatable as to what constitutes acting on a location. Indeed, pointing at a target position and reaching toward one may be functionally equivalent instances of performing a goal-directed action. Welsh et al. (2005) did argue that social IOR was an evolved mechanism concerned with visual search. Specifically, it is uneconomical to search where another individual has just searched. If search efficiency is the critical component then pointing to a location presumably indicates that that location has been examined by 
the pointer. Recall that head and eye gaze alone is sufficient to generate social IOR (Skarratt et al., 2010). The effect may therefore be caused by a range of potential cues, which indicate the location that another person has previously explored. It may therefore be that IOR is itself co-represented as part of joint search activity. It has been shown that higher level processes such as inhibitory control and visual search strategies can be shared across co-participants in the same task (Dale, Kirkham, \& Richardson, 2011; Schuch \& Tipper, 2007). It is possible that some joint corepresentation of attention therefore occurs, eliciting social IOR during joint search.

In contrast to action co-representation, the present data is consistent with the view that the effect has more in common with classical IOR. Like its solitary counterpart, social IOR is sensitive to the object or location attended, rather than the particular response made toward that location (Jordan \& Tipper, 1998; Tipper, Driver, \& Weaver, 1991). Indeed, the present results are consistent with findings within the IOR literature. For instance, it has been shown that IOR is demonstrated to cued locations, when the response is a manual pointing movement (Fischer, Pratt, \& Neggers, 2003). In addition, the data are consistent with work indicating that pointing gestures do automatically orient attention (Langton \& Bruce, 2000), as do other types of central cue including goal directed reaching, as well as eye and head direction (Driver, et al., 1999; Friesen \& Kingstone, 1998; Frischen, Bayliss, \& Tipper, 2007). Furthermore, the abolition of the effect when co-actors are required to make a discrimination judgment also concurs with what is known about IOR (Tanaka \& Shimojo, 1996; Terry, et al., 1994). 
An account of social IOR in terms of attentional/oculomotor reorienting and may help to develop the interpretation of previous findings in the literature. For example, Welsh, et al. (2009b) found that participants with high functioning autistic spectrum disorder (ASD) did not show inhibition when compared to typically developing controls. The authors here suggest that these findings may be as a result of differences in action co-representation between ASD populations and controls, namely mirror neuron dysfunction. Nonetheless, there is much support in the literature for dissociations between ASD and control participants in orienting attention to social cues such as gaze (see Frischen, et al., 2007 for a revew; Ristic, et al., 2005; Senju, Tojo, Dairoku, \& Hasegawa, 2004). Conversely, some evidence speaks against dysfunctions of action co-representation in ASD participants during joint action (Sebanz, Knoblich, Stumpf, \& Prinz, 2005). As Skarratt et al (2010) demonstrated in typically developing populations, both head/eye gaze and action cues are sufficient to observe social IOR. Welsh et al. may therefore have made the novel contribution that ASD participants show different orienting behaviors to both eye gaze and action cues. This interpretation would be consistent with the present findings and a general model of social IOR as a visual orienting phenomenon.

The present work showed that for inhibition, a performed action does not need to be the same as that observed when both are directed to the same side of a participant's visual field. Welsh et al. (2009a) also found inhibitory effects when participants aimed actions toward the same side of visual space to those just observed. An account of social IOR based on orienting may explain the results of both of these 
studies. In both Welsh et al. and in the current data, slowest responses occur when attention must be oriented in the same direction as a previously observed and this action is made to the same target object. Furthermore as in Welsh et al.'s paradigm, some response slowing was present when observed and performed responses were made to the same area of visual space but to different locations, relative to those made towards the opposite side of visual space. Finally, in the present findings, when participants made different kinematic responses to those observed, those made to the same side of visual space were relatively slowed. When these studies are considered together, clearly visual factors are critical in determining whether inhibition is present. They both therefore support an account of social IOR based upon the spatial location of action, rather than the nature of the observed movement.

Whilst behaviorally, social IOR seems to be an IOR-like effect that is socially modulated, a further question remains concerning whether it is subserved by district neural processes. The current findings suggest that similar networks that underlie IOR may generate the effect. In particular the superior colliculus is thought to have a key role in generating individual IOR, alongside structures involved in spatial working memory, which are thought to maintain a mapping of the visual environment in spatiotopic coordinates (e.g., Sumner, Nachev, Vora, Husain, \& Kennard, 2004). Experiment 1, which revealed inhibitory tagging to objects and locations in the response environment indicates that similar structures may contribute to social IOR. 
A partial limitation of the current study, is that only RTs (i.e., the time taken to initiate a movement following the presentation of a stimulus) were measured as an index of social IOR. RTs were appropriate both theoretically, due to the prediction that attentional manipulations would modulate the effect in Experiments 1 and 2 as well as practically because movement latencies of pointing responses could not be measured using the touchscreen in Experiment 3. Previous studies have not demonstrated action slowing effects occurring between the initiation and the execution of responses, indicating that the effect is generated at the perceptuoattentional or response planning stages of processing (Skarratt, et al., 2010; Welsh, et al., 2005; Welsh, et al., 2007; Welsh, et al., 2009b). As we set out above, the current findings are consistent with this interpretation as manipulations of perceptual aspects of the response environment, modulated social IOR. Specifically, Experiment 1 showed that social IOR is sensitive to whether the observed action is performed on the same object as the executed action. This is highly consistent with demonstrations of the same effect in solitary IOR tasks between cue and target. In addition, and also consistent with the IOR literature, Experiment 2 demonstrated that social IOR is limited to localization of targets, rather than discrimination of two stimuli at the SOA used in the current experiments (1300-1700ms). Both of these results concur with the findings of Hayes et al. (2010) who found that slowed responses following sensory transients and another's actions were roughly equivalent. In individual manual aiming paradigms however, when a goal-directed reaching response is made, an identical action to the same location can be delayed in response time yet facilitated in movement time (Tremblay, et al., 2005). Moreover, if social IOR does have a corepresentational component, this may manifest itself in movement times. Additionally, although Experiment 3 of the current study found no RT benefit for 
performing the same manual gesture, a number of studies have identified facilitation for matching performed to an observed goal-directed action by response effector. This manipulation has been found to affect response following action cues in attentional cueing paradigms (Belopolsky, Olivers, \& Theeuwes, 2008). In other work involving transfer of learning however, intra-manual transfer has been shown to be present following action observation using non-mirrored effectors (Hayes, Andrew, Elliott, Roberts, \& Bennett, 2012). The role of response hand remains an important question in the social IOR paradigm.

The literature on joint action makes a distinction between the co-representation of a partner's task and of their action. For example, tasks where spatially compatible stimuli elicit co-representation of another's action can also be modulated when participants either respond to the same or different non-spatial dimensions of the stimuli (Atmaca, et al., 2011; Sebanz, Knoblich, et al., 2005a). Jointly acting participants may therefore co-represent both the action and the task of another. The possibility remains that, even if social IOR is caused by orienting to locations, corepresentation of the other's task may contribute to the effect. For example since the present paradigm is turn-based, its structure clearly indicates when a partner will perform an action and this can be used to prepare participant's own responses. Thus co-representing the partner's task may remain crucial to monitoring another's action and orienting to their responses. 
In sum, the present set of experiments has shown that when a response is observed, goal-directed aiming movements to the same object and location are slowed. This effect is modulated by task factors known to affect performance in individual IOR studies. No evidence was found for inhibitory coding made on the basis of observing a particular action and therefore the co-representation of motor responses. The current findings support an account of social IOR wherein attention is slowed to return to locations that have been responded to by another human agent.

\section{References}

Atmaca, S., Sebanz, N., \& Knoblich, G. (2011). The joint flanker effect: sharing tasks with real and imagined co-actors. Experimental Brain Research, 1-15.

Atmaca, S., Sebanz, N., Prinz, W., \& Knoblich, G. (2008). Action co-representation: The joint SNARC effect. Social neuroscience, 3, 410-420.

Belopolsky, A. V., Olivers, C. N. L., \& Theeuwes, J. (2008). To point a finger: Attentional and motor consequences of observing pointing movements. Acta Psychologica, 128, 56-62.

Blakemore, S. J., \& Frith, C. (2005). The role of motor contagion in the prediction of action. Neuropsychologia, 43, 260-267.

Brass, M., Bekkering, H., \& Prinz, W. (2001). Movement observation affects movement execution in a simple response task. Acta Psychologica, 106, 3-22.

Brass, M., Bekkering, H., Wohlschlager, A., \& Prinz, W. (2000). Compatibility between observed and executed finger movements: comparing symbolic, spatial, and imitative cues. Brain and cognition, 44, 124-143. 
Calvo-Merino, B., Glaser, D. E., Grezes, J., Passingham, R. E., \& Haggard, P. (2005). Action observation and acquired motor skills: an FMRI study with expert dancers. Cerebral cortex, 15, 1243-1249.

Cole, G. G., Skarratt, P. A., \& Billing, R. C. (2012). Do action goals mediate social inhibition of return? Psychological research, 1-11.

Dale, R., Kirkham, N. Z., \& Richardson, D. C. (2011). The Dynamics of Reference and Shared Visual Attention. Frontiers in psychology, 2, 355.

di Pellegrino, G., Fadiga, L., Fogassi, L., Gallese, V., \& Rizzolatti, G. (1992). Understending motor events: a neurphysisiological study. Experimental Brain Research, 91, 176-180.

Dolk, T., Hommel, B., Colzato, L. S., Schütz-Bosbach, S., Prinz, W., \& Liepelt, R. (2011). How social is the social Simon effect? Frontiers in psychology, 2, 84.

Driver, J., Davis, G., Ricciardelli, P., Kidd, P., Maxwell, E., \& Baron-Cohen, S. (1999). Gaze perception triggers reflexive visuospatial orienting. Visual Cognition, 6, 509-540.

Duncan, J. (1984). Selective attention and the organization of visual information. Journal of Experimental Psychology: General, 113, 501.

Fabbri-Destro, M., \& Rizzolatti, G. (2008). Mirror neurons and mirror systems in monkeys and humans. Physiology, 23, 171-179.

Filimon, F., Nelson, J., \& Hagler, D. (2007). Human cortical representations for reaching: mirror neurons for execution, observation, and imagery. Neuroimage, 22, 629.

Fischer, M. H., Pratt, J., \& Neggers, S. F. W. (2003). Inhibition of return and manual pointing movements. Perception, \& Psychophysics, 65, 379-387. 
Fogassi, L., Ferrari, P. F., Gesierich, B., Rozzi, S., Chersi, F., \& Rizzolatti, G. (2005). Parietal lobe: from action organization to intention understanding. Science, 308(5722), 662-667.

Friesen, C. K., \& Kingstone, A. (1998). The eyes have it! Reflexive orienting is triggered by nonpredictive gaze. Psychonomic Bulletin \& Review, 5, 490-495.

Frischen, A., Bayliss, A. P., \& Tipper, S. P. (2007). Gaze cueing of attention: visual attention, social cognition, and individual differences. Psychological bulletin, $133,694$.

Frischen, A., Loach, D., \& Tipper, S. P. (2009). Seeing the world through another person's eyes: Simulating selective attention via action observation. Cognition, $111,212-218$.

Gallese, V., Fadiga, L., Fogassi, L., \& Rizzolatti, G. (1996). Action recognition in the premotor cortex. Brain, 119, 593-609.

Hayes, S. J., Andrew, M., Elliott, D., Roberts, J. W., \& Bennett, S. J. (2012). Dissociable contributions of motor-execution and action-observation to intermanual transfer. Neuroscience letters, 506, 346-350.

Hayes, S. J., Hansen, S., \& Elliott, D. (2010). Between-person effects on attention and action: Joe and Fred revisited. Psychological research, 74, 302-312.

Hommel, B. (2009). Action control according to TEC (theory of event coding). Psychological research, 73, 512-526.

Howard, L. A., Lupáñez, J., \& Tipper, S. P. (1999). Inhibition of return in a selective reaching task: An investigation of reference frames. The Journal of general psychology, 126, 421-442. 
Iacoboni, M., Molnar-Szakacs, I., Gallese, V., Buccino, G., Mazziotta, J. C., \& Rizzolatti, G. (2005). Grasping the intentions of others with one's own mirror neuron system. PLoS Biology, 3, e79.

Jeannerod, M., \& Frak, V. (1999). Mental imaging of motor activity in humans. Current opinion in neurobiology, 9, 735-739.

Johnson-Frey, S. H., Maloof, F. R., Newman-Norlund, R., Farrer, C., Inati, S., \& Grafton, S. T. (2003). Actions or hand-object interactions? Human inferior frontal cortex and action observation. Neuron, 39, 1053-1058.

Jordan, H., \& Tipper, S. P. (1998). Object-based inhibition of return in static displays. Psychonomic Bulletin \& Review, 5, 504-509.

Jordan, H., \& Tipper, S. P. (1999). Spread of inhibition across an object's surface. British Journal of Psychology, 90, 495-507.

Kilner, J. M., Paulignan, Y., \& Blakemore, S. J. (2003). An Interference Effect of Observed Biological Movement on Action. Current Biology, 13, 522-525.

Kingstone, A., \& Pratt, J. (1999). Inhibition of return is composed of attentional and oculomotor processes. Attention, Perception, \& Psychophysics, 61, 10461054.

Klein, R. M. (2000). Inhibition of return. Trends in Cognitive Sciences, 4, 138-147.

Knoblich, G., \& Sebanz, N. (2006). The social nature of perception and action. Current Directions in Psychological Science, 15, 99-104.

Kwak, H., \& Egeth, H. (1992). Consequences of allocating attention to locations and to other attributes. Attention, Perception, \& Psychophysics, 51, 455-464.

Langton, S. R., \& Bruce, V. (2000). You must see the point: automatic processing of cues to the direction of social attention. Journal of experimental psychology. Human perception and performance, 26, 747-757. 
Lyons, J., Weeks, D. J., \& Elliott, D. (2013). The Gambler's Fallacy: A Basic Inhibitory Process? Frontiers in psychology, 4, 72.

Mukamel, R., Ekstrom, A. D., Kaplan, J., Iacoboni, M., \& Fried, I. (2010). SingleNeuron Responses in Humans during Execution and Observation of Actions. Current Biology, 20, 750-756.

Ondobaka, S., de Lange, F. P., Newman-Norlund, R. D., Wiemers, M., \& Bekkering, H. (2012). Interplay between action and movement intentions during social interaction. Psychological Science, 23, 30-35.

Posner, M. I., \& Cohen, Y. (1984). Components of Visual Orienting. In H. Bouma \& D. G. Bouwhuis (Eds.), Attention and performance X (pp. 531-556): Erlbaum.

Prinz, W. (1997). Perception and action planning. European journal of cognitive psychology, 9, 129-154.

Rafal, R., Calabresi, P. a., Brennan, C. W., \& Sciolto, T. K. (1989). Saccade preparation inhibits reorienting to recently attended locations. Journal of experimental psychology. Human perception and performance, 15, 673-685.

Ristic, J., Mottron, L., Friesen, C. K., Iarocci, G., Burack, J. A., \& Kingstone, A. (2005). Eyes are special but not for everyone: The case of autism. Cognitive Brain Research, 24, 715-718.

Rizzolatti, G., \& Craighero, L. (2004). The mirror-neuron system. Annual review of neuroscience, 27, 169-192.

Schuch, S., \& Tipper, S. P. (2007). On observing another person's actions: Influences of observed inhibition and errors. Perception \& Psychophysics, 69, 828.

Sebanz, N., Knoblich, G., \& Prinz, W. (2003). Representing others' actions: Just like one's own? Cognition, 88, B11-B21. 
Sebanz, N., Knoblich, G., \& Prinz, W. (2005a). How two share a task: corepresenting stimulus-response mappings. Journal of Experimental Psychology: Human Perception and Performance, 31, 1234.

Sebanz, N., Knoblich, G., \& Prinz, W. (2005b). How two share a task: corepresenting stimulus-response mappings. Journal of experimental psychology. Human perception and performance, 31, 1234-1246.

Sebanz, N., Knoblich, G., Prinz, W., \& Wascher, E. (2006). Twin peaks: An ERP study of action planning and control in coacting individuals. Journal of Cognitive Neuroscience, 18, 859-870.

Sebanz, N., Knoblich, G., Stumpf, L., \& Prinz, W. (2005). Far from action-blind: Representation of others' actions in individuals with autism. Cognitive Neuropsychology, 22, 433-454.

Senju, A., Tojo, Y., Dairoku, H., \& Hasegawa, T. (2004). Reflexive orienting in response to eye gaze and an arrow in children with and without autism. Journal of Child Psychology and Psychiatry, 45, 445-458.

Simon, J. (1970). Effects of an irrelevant auditory stimulus on visual choice reaction time. Journal of Experimental Psychology, 86, 272-274.

Skarratt, P. A., Cole, G. G., \& Kingstone, A. (2010). Social inhibition of return. Acta Psychologica, 134, 48-54.

Skarratt, P. A., Cole, G. G., \& Kuhn, G. (2012). Visual cognition during real social interaction. Frontiers in Human Neuroscience, 6, 196.

Stürmer, B., Aschersleben, G., \& Prinz, W. (2000). Correspondence effects with manual gestures and postures: a study of imitation. Journal of Experimental Psychology: Human Perception and Performance, 26, 1746. 
Sumner, P., Nachev, P., Vora, N., Husain, M., \& Kennard, C. (2004). Distinct cortical and collicular mechanisms of inhibition of return revealed with $\mathrm{S}$ cone stimuli. Current Biology, 14, 2259-2263.

Tanaka, Y., \& Shimojo, S. (1996). Location vs feature: Reaction time reveals dissociation between two visual functions. Vision Research, 36, 2125-2140.

Terry, K. M., Valdes, L. A., \& Neill, W. T. (1994). Does inhibition of return occur in discrimination tasks? Attention, Perception, \& Psychophysics, 55, 279-286.

Tipper, S. P., Driver, J., \& Weaver, B. (1991). Short report: Object-centred inhibition of return of visual attention. The Quarterly Journal of Experimental Psychology, 43, 289-298.

Tipper, S. P., Jordan, H., \& Weaver, B. (1999). Scene-based and object-centered inhibition of return: Evidence for dual orienting mechanisms. Attention, Perception, \& Psychophysics, 61, 50-60.

Tremblay, L., Welsh, T. N., \& Elliott, D. (2005). Between-trial inhibition and facilitation in goal-directed aiming: manual and spatial asymmetries. Experimental Brain Research, 160, 79-88.

Tsai, C.-C., Sebanz, N., \& Knoblich, G. (2011). The GROOP effect: Groups mimic group actions. Cognition, 118, 135-140.

Villiger, M., Chandrasekharan, S., \& Welsh, T. N. (2011). Activity of human motor system during action observation is modulated by object presence. Experimental Brain Research, 209, 85-93.

Welsh, T. N., Elliott, D., Anson, J. G., Dhillon, V., Weeks, D. J., Lyons, J. L., \& Chua, R. (2005). Does Joe influence Fred's action? Inhibition of return across different nervous systems. Neuroscience letters, 385, 99-104. 
Welsh, T. N., Lyons, J., Weeks, D. J., Anson, J. G., Chua, R., Mendoza, J., \& Elliott, D. (2007). Within- and between-nervous-system inhibition of return: observation is as good as performance. Psychonomic Bulletin \& Review, 14, 950-956.

Welsh, T. N., McDougall, L. M., \& Weeks, D. J. (2009a). The performance and observation of action shape future behaviour. Brain and cognition, 71, 64-71.

Welsh, T. N., \& Pratt, J. (2006). Inhibition of return in cue-target and target-target tasks. Experimental Brain Research, 174, 167-175.

Welsh, T. N., Ray, M. C., Weeks, D. J., Dewey, D., \& Elliott, D. (2009b). Does Joe influence Fred's action? Not if Fred has autism spectrum disorder. Brain research, 1248, 141-148.

Wilson, M., \& Knoblich, G. (2005). The case for motor involvement in perceiving conspecifics. Psychological bulletin, 131, 460-473. 


\section{Figure Captions}

Figure 1: The response environment. Participants are depicted with their preferred hand resting in the "home" position, before they alternated responses to targets appearing in the left or right positions. Measurements are not drawn to scale.

Figure 2: Stimulus positions in Experiment 1.

Figure 3; Mean RTs to initiate responses to targets as a function of object type and their position relative to partner's prior response, in Experiment 1. Standard error of the mean bars are included. 
Figure 4: Mean RTs to initiate responses to targets as a function of task type and their position relative to partner's prior response, in Experiment 2. Standard error bars are included.

Figure 5: Mean reaction times for initiation toward target locations across each condition of action performed by participants in relation to those of partners. Error bars represent the standard error of the mean. 


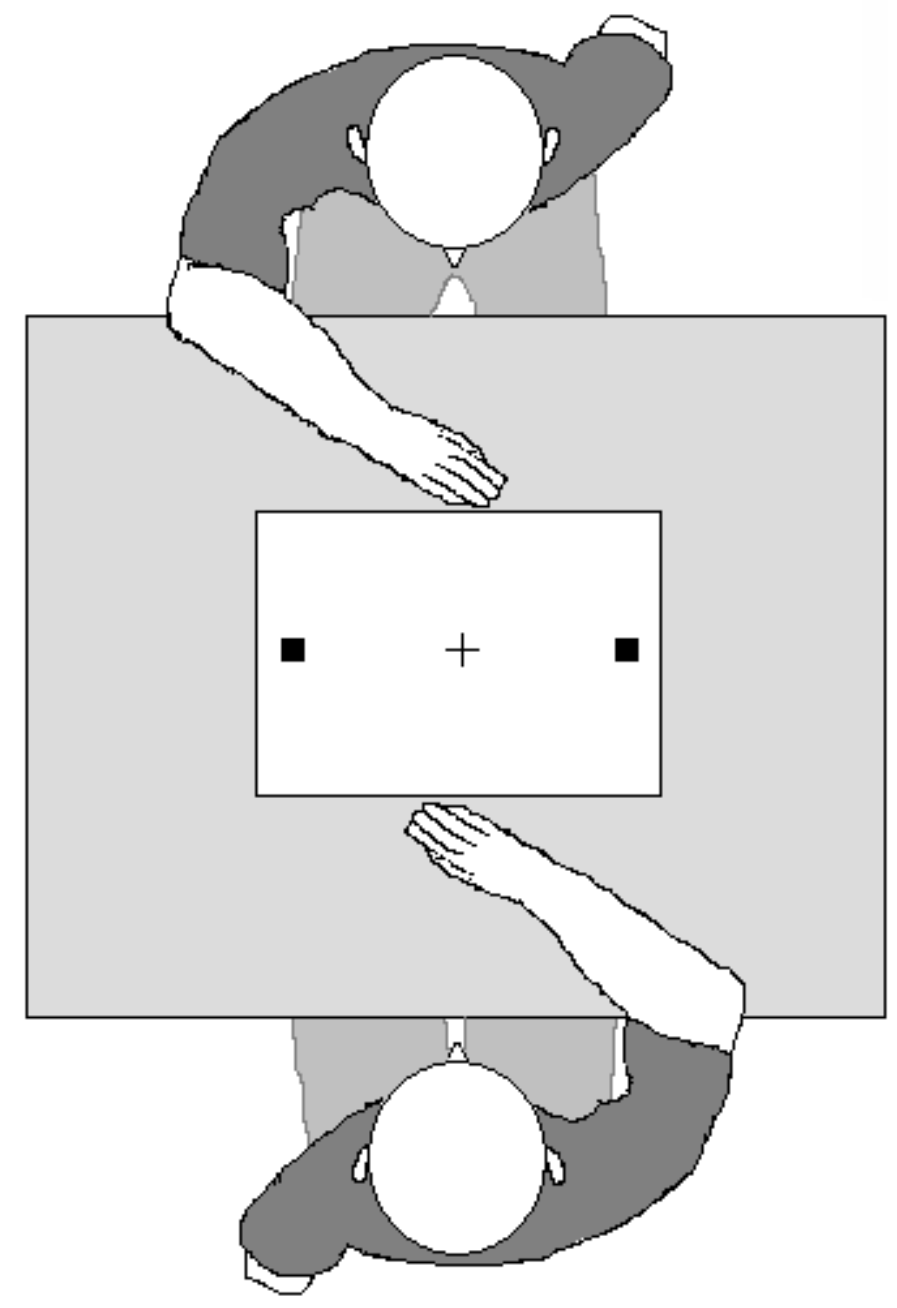

Figure 1 
Single static

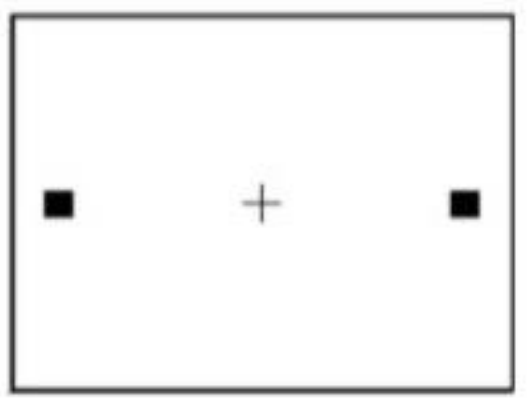

Single moving

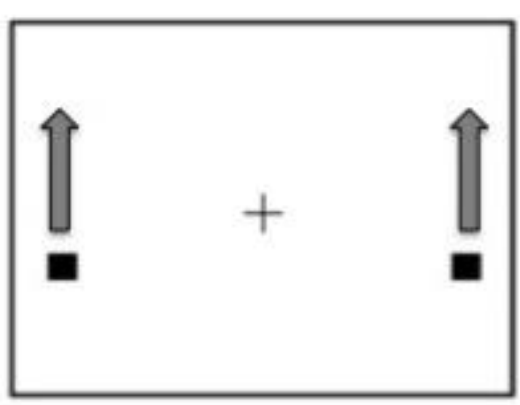

Displaced grouped

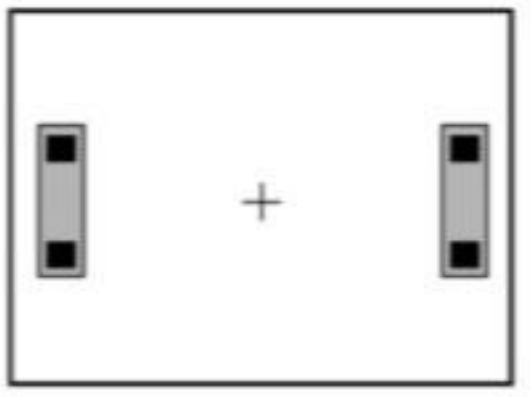

Displaced ungrouped

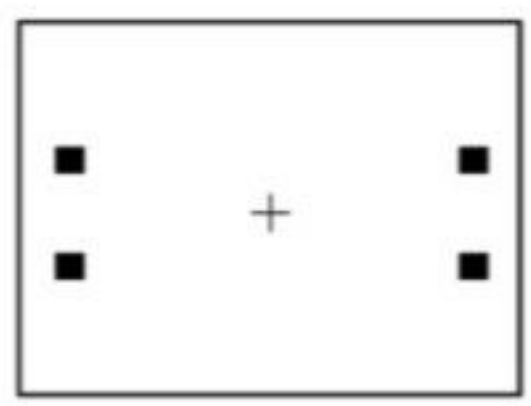

Figure 2 


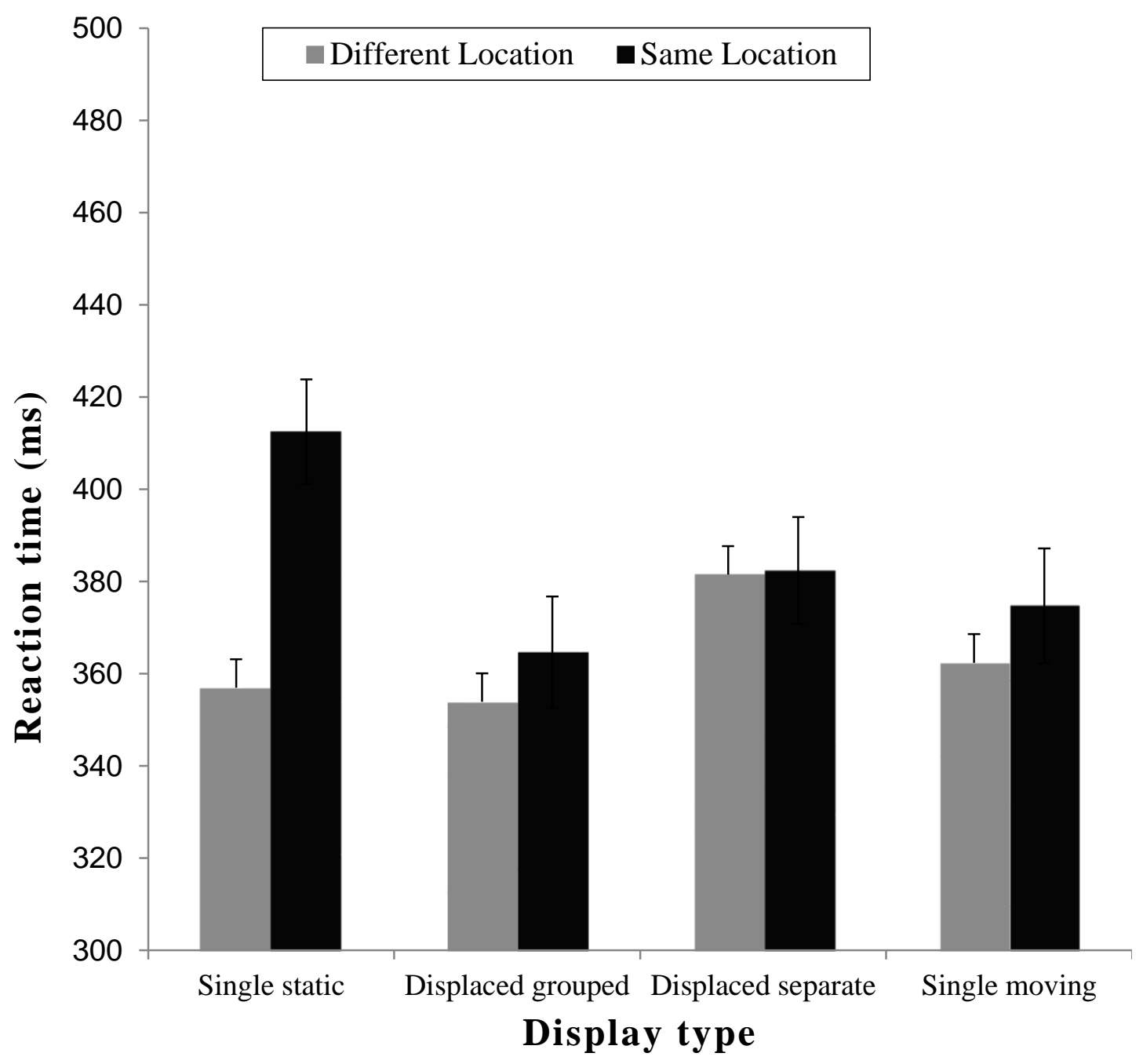

Figure 3 


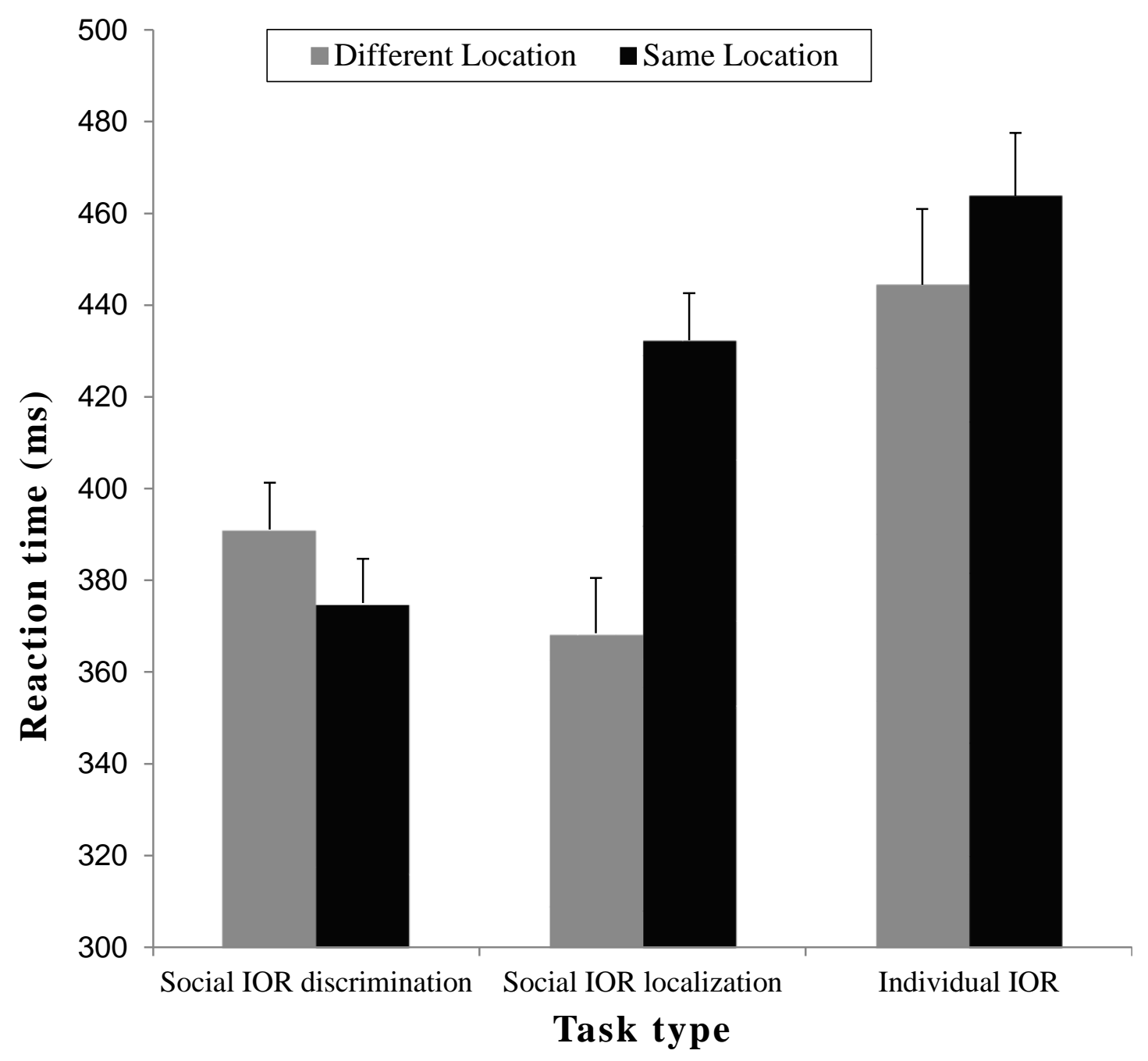

Figure 4 


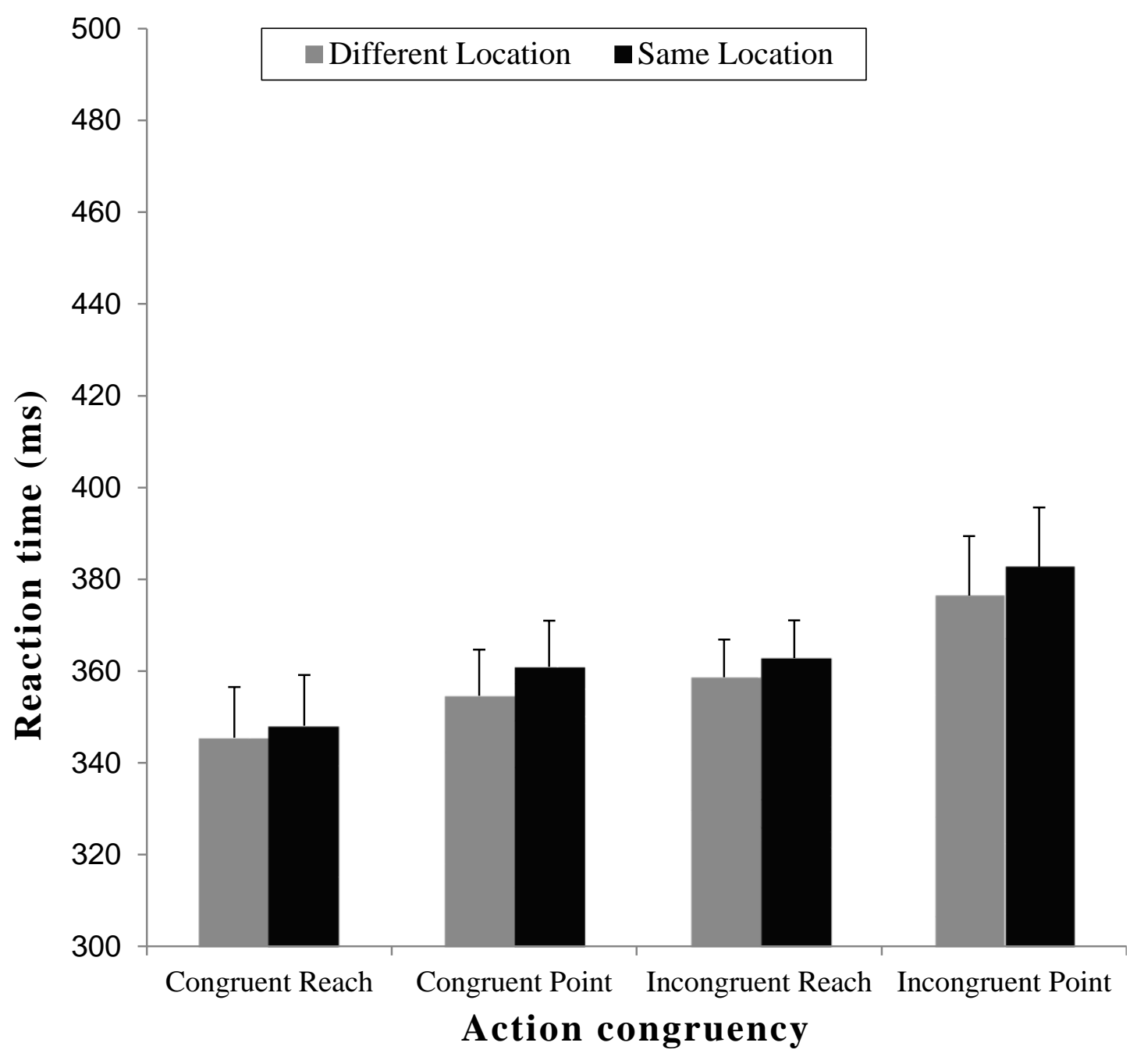

Figure 5 